\title{
Tisser le texte et cacher les fils : l'écriture plurilingue de Manuel Puig
}

\section{Delfina Cabrera}

\section{(2) OpenEdition}

12 Journals

\section{Édition électronique}

URL : http://journals.openedition.org/genesis/2625

DOI : 10.4000/genesis.2625

ISSN : 2268-1590

\section{Éditeur :}

Presses universitaires de Paris Sorbonne (PUPS), Société internationale de génétique artistique littéraire et scientifique (SIGALES)

\section{Édition imprimée}

Date de publication : 4 juin 2018

Pagination : 51-63

ISBN : 979-10-231-0604-6

ISSN : 1167-5101

\section{Référence électronique}


Tisser le texte et cacher les fils : l'écriture plurilingue de Manuel Puig

Delfina Cabrera

Trahir la langue.

Le plurilinguisme d'un écrivain pop

$$
\begin{array}{r}
\text {-Avec qui partagez-vous un style? } \\
\text { - Je suis désolé, je ne connais pas grand-chose à la littérature. } \\
\text { Manuel PUIG }{ }^{1}
\end{array}
$$

Émigré, nomade, cosmopolite, exilé, déporté, expatrié... C'est ainsi qu'on a souvent désigné l'écrivain argentin Manuel Puig (1932-1990) lors des tentatives de saisir l'étrangeté inhérente à son œuvre et à sa trajectoire d'auteur. De fait, bien que depuis quelques décennies ses romans fassent partie du canon de la littérature latino-américaine (en 1982, Puig fut même proposé comme candidat au prix Nobel), sa reconnaissance par la critique n'a pas été immédiate. Son travail avec la culture de masse, de même que sa rupture avec les traits principaux du boom latino-américain ${ }^{2}$ ont contribué à forger sa réputation d'auteur étrange, voire étranger, au champ littéraire qui lui était contemporain.

En 1968, Puig fait irruption dans la littérature argentine avec La traición de Rita Hayworth [La Trahison de Rita Hayworth], un roman qui prenait comme source primaire des matériaux dédaignés par la culture littéraire traditionnelle - le mélodrame, le cinéma hollywoodien des années 1930 et 1940, le pop art, le tango, le boléro, le feuilleton - démarche impossible à faire accepter par une tradition savante dont Jorge Luis Borges était le référent incontestable ${ }^{3}$. Mais la nouveauté apportée par Puig ne consistait pas seulement en l'incorporation de l'industrie culturelle dans l'institution littéraire; bien au contraire, La traición de Rita Hayworth opérait une désacralisation de «La Littérature» avec un L majuscule 4 . Sous l'apparence d'un langage simple, voire banal - incarné notamment dans de longs dialogues et monologues intérieurs présentés sans aucune intervention d'un narrateur ${ }^{5}-$, demeurait une écriture audacieuse et expérimentale. La réception mitigée de

Genesis 46, 2018 son opéra prima peut sans doute s'expliquer par le fait que l'acte de création de Puig était incontestablement un acte de transgression par rapport à la définition traditionnelle de la littérature :

...nous avons été obligés de penser que Puig était un écrivain «populaire et périphérique»; mais penser dans ces termes

1. «Manuel Puig y el paraíso perdido », entretien avec Manuel Puig réalisé par Jorgelina Corbatta en 1979. Dans Julia Romero, Puig por Puig. Imágenes de un escritor, Frankfurt am Main/Madrid, IberoamericanaVervuert-Verlag, 2006, p. 294. Notre traduction.

2. Le boom latino-américain est un mouvement littéraire et un phénomène éditorial des années 1960 et 1970, défini par la publication et diffusion en Europe, Amérique Latine et les États-Unis des romans d'un groupe d'écrivains latino-américains associés au « réalisme magique », notamment Gabriel García Márquez, Julio Cortázar, Carlos Fuentes, Juan Carlos Onetti, Mario Vargas Llosa et Alejo Carpentier. Néanmoins, la définition du phénomène continue à susciter des débats. Pour une analyse critique du boom voir Ángel Rama, Más allá del boom. Literatura y mercado, Buenos Aires, Folios, 1984 ; Noé Jitrik (dir.), La narración gana la partida Historia crítica de la literatura argentina, vol. XI, Buenos Aires, Emecé, 2000 ; Idelber Avelar, Alegorías de la derrota. La ficción pósdictatorial y el trabajo del duelo, Santiago de Chile, Cuarto Propio, 2000 ; Jean Franco, Decadencia y caída de la ciudad letrada, Madrid, Debate, 2003.

3.Selon l'écrivain et critique brésilien Silviano Santiago, Puig « est le premier grand auteur latino-américain qui travaille avec les débris dérivés de l'excès d'excès de l'industrie culturelle américaine et argentine, c'est-à-dire, avec presque des déchets ». Voir Silviano Santiago, « Manuel Puig: a atualidade do precursor», dans Ora (direis) puxar conversa!: ensaios literários, Belo Horizonte, Editora UFMG, 2006, p. 370 (ici et plus loin notre traduction).

4. Jean-Marie Schaeffer, Petite écologie des études littéraires : pourquoi et comment étudier la littérature?, Vincennes, Thierry Marchaisse, 2011, p. 14-15.

5. «Je sais comment parlent les personnages de Puig, mais je ne sais pas comment écrit Puig, je ne connais pas son style », déclara ironiquement Juan Carlos Onetti en 1973 (Juan Carlos Onetti, dans Osvaldo Soriano, Soriano por Soriano, Seix Barral, Buenos Aires, p. 56). 
serait comme penser que Kafka, Warhol, Pasolini ou Copi étaient des artistes populaires et périphériques. Dans tous ces cas, il s'agit d'expériences «non-centrales » (car, précisément, ils disqualifient la notion de «centre» dérivée des dispositifs des systèmes de catégorisation); mais surtout, il s'agit d'expériences décisives par rapport à ce qu'on pourrait appeler «dissidence» : ce sont des expériences «centrales» de certaines formes de la dissidence 6 .

Or, chez Puig, plus que chez n'importe quel autre auteur argentin de son époque, cette dissidence s'ancre aussi dans une zone de son œuvre rarement étudiée : son écriture plurilingue. De fait, comme il ressort du témoignage de Daniel Link ci-dessus, Puig s'inscrit dans une lignée d'écrivains «périphériques » comme Franz Kafka, Pier Paolo Pasolini ou Copi ; des auteurs qui ont eu, d'ailleurs, eux aussi, plusieurs langues d'écriture. Paradoxalement, le plurilinguisme de Puig n'est pas pris en compte quand il s'agit d'analyser la singularité qui caractérise sa production littéraire.

En effet, à quelques exceptions près qui seront évoquées plus loin, les critiques ont généralement laissé en marge la nature hétérolingue du processus créatif chez Puig en se concentrant sur d'autres problématiques comme la déstabilisation des genres narratifs, la tension entre la culture des élites et la culture populaire, l'absence du narrateur omniscient ou encore les questions de sexualité et de genre. Les causes de cet oubli critique peuvent être de plusieurs ordres, mais une chose est sûre, les textes plurilingues de Puig ne respectent pas une norme essentielle du champ littéraire latino-américain : l'usage de l'espagnol en tant que langue littéraire légitime et unique. Comme le précise Roberto Ignacio Díaz :

Si le monolinguisme du continent n'était pas une tautologie enracinée, il n'y aurait pas besoin de souligner le fait que l'Amérique espagnole n'a pas un véritable centre linguistique et qu'elle est clairement une région polyglotte. [...] Les institutions de la littérature - les auteurs de même que les historiens et critiques littéraires - ont également collaboré à la consécration virtuelle de la langue espagnole non seulement comme lingua franca (ce qu'elle est, faute de mieux), mais comme le seul (et inhérent) instrument utile pour avoir une tradition littéraire latino-américaine unie?.

Le boom des années 1960 et 1970 a en effet contribué à propager la vision d'une littérature latino-américaine engagée dont les œuvres devaient être écrites en langue espagnole, symbole et repère identitaires forts d'une communauté latino-américaine qui existerait au-delà des frontières nationales et de celles du continent. Pour les écrivains latino-américains de l'époque, écrire en une «langue autre» impliquait, d'une part, un éloignement de cette communauté «pan-latinoaméricaine» et, d'autre part, une confrontation avec des questionnements socio-historiques plus vastes dont l'origine remontait à la formation des États-Nations et à l'affirmation des langues nationales qui l'ont accompagnée8. Or Puig fut l'un des rares auteurs à prendre ce risque en faisant recours à des stratégies créatives qui appellent à la cohabitation, à la recomposition et à la transformation mutuelle des langues. Dans ce contexte, même si les textes publiés de Puig ont globalement une apparence monolingue, c'est précisément cette «prétendue homogénéité» qu'il faut questionner en s'appuyant sur l'approche génétique.

Comme c'est le cas pour de nombreux auteurs plurilingues, l'impact du plurilinguisme sur le processus créatif de Puig n'est pas visible dans ses textes publiés, ce qui expliquerait en partie pourquoi les critiques et le grand public ont ignoré cette particularité de son œuvre. Pourtant, l'écriture en plusieurs langues, qu'on peut observer notamment dans les étapes initiales du travail créatif de l'écrivain, est en grande partie responsable de la «trahison» de la langue littéraire normée qu'on lui a souvent reprochée. Mais celle-ci ne peut être comprise qu'en analysant ses documents de travail : des brouillons, des notes, des schémas et des croquis. Plus de vingt mille documents - et plus de trois mille films en VHS - qui composent les Archives Puig9 permettent de briser toute illusion d'une écriture linguistiquement homogène et fidèle

6. Daniel Link, « Kitsch, camp, boom: Puig y el ser moderno », conférence prononcée le 18 septembre 2013 à l'université de l'État de Rio de Janeiro (UERJ). Le texte est disponible en ligne : <http://www.academia. edu/6458921/_Kitsch_camp_boom_Puig_y_el_ser_moderno_Daniel_ Link_> (consulté en décembre 2017).

7. Ignacio Roberto Díaz, Unhomely Rooms: Foreign Tongues and Spanish American Literature, Lewisburg, Pennsylvania, Bucknell University Press, 2002, p. 33-34.

8. Pour approfondir cette question, voir Juan Antonio Ennis, Decir la lengua. Debates ideológico-lingüísticos en Argentina desde 1837, Frankfurt am Main, Peter Lang, 2008.

9. Malgré ses déplacements continus - tout au long de sa vie, il vécut dans au moins cinq pays différents : Argentine, Italie, Mexique, États-Unis, Brésil -, Puig a conservé la plupart des manuscrits qui composent 
au paradigme monolingue10. Certes, Puig était un écrivain pop, mais surtout dans le sens que Gilles Deleuze et Felix Guattari donnent à ce terme dans Kafka, pour une littérature mineure : «Une issue pour le langage, pour la musique, pour l'écriture. Ce qu'on appelle Pop - Pop'musique, Pop'philosophie, Pop'écriture : Wörterflucht. Se servir du polylinguisme dans sa propre langue, faire de celle-ci un usage mineur ou intensif 11 ...». Autrement dit, il s'agit pour les créateurs qui se sont engagés dans cette démarche d'arriver à s'inventer activement un style en creusant l'unicité de l'expérience monolingue. Tout comme celui de José María Arguedas, Guillermo Cabrera Infante, Rodolfo Wilcock ou Sylvia Molloy, Alejandra Pizarnik (quelques écrivains latinoaméricains plurilingues parmi tant d'autres), le style de Puig appartient à ceux qui n'ont pas de style ou bien à ceux qui construisent une langue étrangère dans leur propre langue.

C'est grâce au cinéma, une passion qui l'habite depuis son enfance, que Puig établit un rapport singulier avec des langues autres que l'espagnol du Río de La Plata. Né au sein d'une famille de classe moyenne à General Villegas, un petit village perdu au milieu de la pampa - un «village de Western», selon ses propres mots ${ }^{12}$, à l'âge de 3 ans il assiste, fasciné, à la projection du film La Fiancée de Frankenstein 13 et devient un cinéphile passionné. La salle de cinéma sera dès lors son échappatoire d'une réalité «teintée de pampa et de machisme ${ }^{14}$ ». Les films, étrangers la plupart du temps, deviendront l'une des sources principales de son imagination et enrichiront une sensibilité plurilingue qui était déjà éveillée en lui par le contact avec le dialecte italien de Parma-Piacenza qu'il avait hérité de ses grands-parents immigrés. Pendant sa jeunesse, tout en poursuivant son rêve de devenir réalisateur, il fait des études d'anglais, ainsi que d'italien, d'allemand et de français dans des instituts de langue (Dante Alighieri, Goethe Institut, Alliance Française). Il apprendra aussi le portugais une fois qu'il sera installé au Brésil dans les années 1980.

Cette pluralité linguistique se manifeste dans le processus créatif de Puig dès les premiers textes dont les avant-textes sont conservés dans les archives : Ball Cancelled (1956) et Summer Indoors (1959), deux scénarios pour le cinéma, écrits à la fois en anglais et en espagnol. La présence des langues étrangères dans les premières œuvres de Puig nous oblige à repenser la vision du plurilinguisme de l'auteur comme étant liée principalement à des facteurs externes, et notamment à l'exil, idée que semblent défendre les quelques rares études consacrées à la présence d'autres langues que l'espagnol dans ses textes. En outre, la majorité de ces études envisage le plurilinguisme de Puig principalement dans la perspective de la traduction et de l'autotraduction 15 - nous y reviendrons.

Cependant, l'usage des langues dans le processus créatif de Puig se révèle beaucoup plus complexe et dépasse le seul cadre de l'écriture (auto)traductive. Olga Anokhina distingue plusieurs stratégies créatives mises en œuvre par des écrivains plurilingues : la séparation fonctionnelle des langues, le code-switching, l'écriture parallèle en deux langues et

maintenant les Archives Puig, parmi les archives d'écrivain les plus complètes d'Argentine. En 1990, lorsque la famille a offert les archives à l'université de La Plata, une équipe de chercheurs a commencé un travail inédit en Argentine : l'analyse d'un fonds important de documents de travail d'un écrivain qui a été suivie de sa numérisation complète et de sa récente mise en ligne sur le site Arcas : <http://arcas.fahce.unlp.edu. ar:8383/greenstone3/library/collection/puig/browse/CL1" \1 "CL1.5"> (consulté en décembre 2017).

10. Selon Yasemin Yildiz, depuis la fin du XVIII siècle, les pratiques plurilingues en tous genres ont été éclipsées par une approche monolingue de la culture et la société. Sous le « paradigme monolingue », les individus, de même que les sociétés ne possèdent « qu'une "vraie" langue, leur "langue maternelle", et cette possession les lie organiquement à une ethnie, une culture et une nation exclusives et clairement délimitées »: Yasemin Yildiz, Beyond the Mother Tongue, New York, Fordham University Press, 2012, p. 2. Notre traduction.

11. Gilles Deleuze et Felix Guattari, Kafka, pour une littérature mineure, Paris, Les Éditions de Minuit, 1975, p. 32.

12. Déclarations de Puig lors d'un entretien accordé à l'émission «A fondo » de la Télévision Espagnole, en 1977.

13. La Fiancée de Frankenstein (titre original : Bride of Frankenstein), James Whale, 1935

14. Entretien accordé à Mario Szulchman dans Julia Romero, Puig por Puig. Imágenes de un escritor, Frankfurt am Main/Madrid, Iberoamericana-Vervuert-Verlag, 2006, p. 160. Ma traduction.

15. Voir Andreia do Santos Menezes, « Manuel Puig: (auto) traductor », dans Xosé Manuel Dasilva et Helena Tanqueiro (dir.), Aproximaciones a la autotraducción, Vigo, Academia del Hispanismo, 2011, p. 141-152; Ilse Logie et Julia Romero, « Extranjeridad: lengua y traducción en la obra de Manuel Puig », Revista Iberoamericana, vol. LXXIV, n 222, 2008, p. 33-51 ; Christopher Larkosh, «Writing in the Foreign: Migrant Sexuality and Translation of the Self in Manuel Puig's Later Work », dans The Translator, $\mathrm{n}^{\circ}$ 12, 2006, p. 279-299; Rossana Álvarez et Juan Ariel Gómez, «Manuel Puig y la estrategia de la (auto)traducción », communication présentée au IIe Congrès CELEHIS de Littérature, Mar del Plata, Argentine, 2004, disponible en ligne : <http://www.mdp.edu.ar/humanidades/letras/ celehis/congreso/2004/actas/ind_titulo.htm> (consulté en décembre 2017); Paula Siganevich, « Brasileridad, traducción y género en la escritura de Manuel Puig », dans José Amícola et Graciela Speranza (dir.), Encuentro Internacional Manuel Puig, Rosario, Beatriz Viterbo, 1998, p. 237-242. 
l'autotraduction ou l'écriture consécutive16. Généralement un écrivain plurilingue privilégie l'une de ces stratégies dans son processus créatif, mais Manuel Puig semble constituer un cas à part, car il utilise l'ensemble de ces stratégies, parfois même au cours de la genèse d'une seule œuvre.

\section{L'utilisation des langues étrangères chez Puig}

L'éventail des stratégies de création plurilingues que nous avons pu observer chez Puig est complexe et varié. Dès lors, établir une typologie exhaustive et représentative de l'usage qu'il fait des langues étrangères devient une tâche peu aisée. De plus, ces stratégies ne se manifestent pas de manière isolée, car chez Puig il y a souvent un va-et-vient continuel entre elles, un passage de l'une à l'autre à des moments différents de l'écriture. Toutefois, malgré cette diversité, une tendance générale peut être identifiée : les langues étrangères sont particulièrement requises pendant l'étape pré-rédactionnelle et participent à l'acte créatif dès la préparation de l'œuvre.

\section{Le code-switching utilisé à l'étape pré-rédactionnelle}

Un phénomène qui semble être particulièrement répandu et que nous avons pu observer surtout à l'étape pré-rédactionnelle du processus créatif est le mélange de l'espagnol avec l'anglais - auquel peuvent s'ajouter ponctuellement d'autres langues, comme le français, l'italien et le portugais. Cette alternance codique ou code-switching caractérise les pratiques langagières courantes des locuteurs plurilingues et constitue un signe distinctif des littératures au carrefour de plusieurs langues ${ }^{17}$ - comme la littérature chicana $^{18}$ et la littérature antillaise -, mais elle prend chez l'argentin Puig une autre forme radicalement différente : celle d'une méthode de composition qui se manifeste lors de la planification.

L'observation des documents de travail de l'auteur, conservés tout au long de sa vie, montre que le codeswitching entre l'espagnol et l'anglais est très présent dans les commentaires métatextuels (fig. 1) :

Importante : No puede haber alivio en $\neq / /$ porque si no

He can go on Forever (what if he does?)

[Important: Il ne peut pas y avoir du soulagement en $\neq / /$ car s'il y en a

Il peut continuer ainsi Pour toujours (que se passerait-il s'il le fait?)] ${ }^{19}$

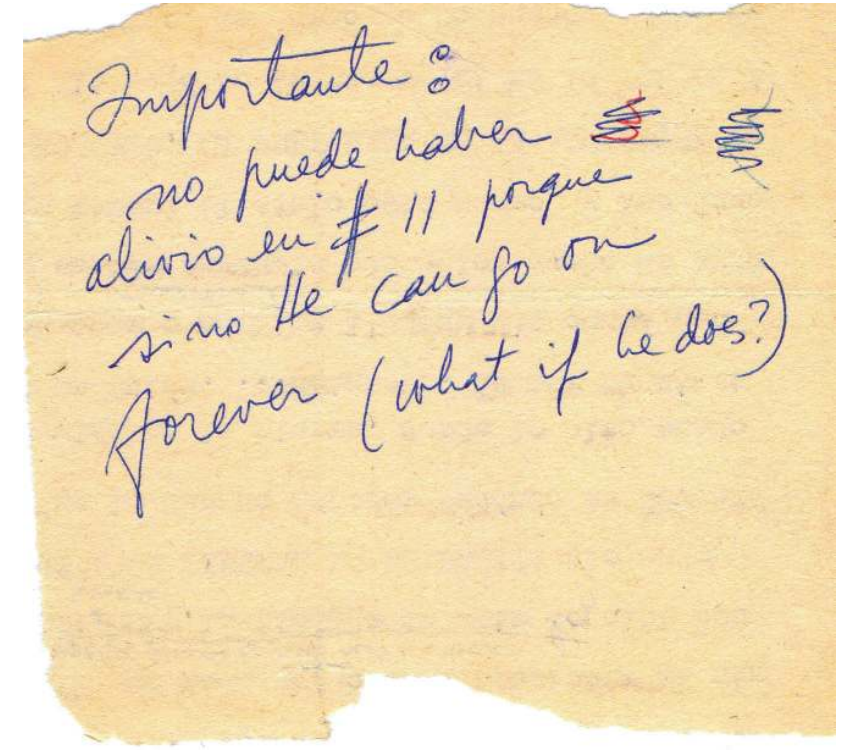

Fig. 1 : Brouillon du roman The Buenos Aires Affair (1973)

Selon le cadre théorique proposé par Almuth Grésillon ${ }^{20}$ et Olga Anokhina ${ }^{21}$, ce mélange de langues peut être comparé à un langage intérieur que l'écrivain utilise dans les documents destinés exclusivement à son usage personnel. Par conséquent, le degré de cohérence de cette écriture est très limité pour des lecteurs externes, tel que nous pouvons

16. Olga Anokhina, «Étudier les écrivains plurilingues grâce aux manuscrits », dans Olga Anokhina et François Rastier (dir.), Écrire en langues : littératures et plurilinguisme, Paris, Éditions des archives contemporaines, coll. «Multilinguisme, traduction, création », 2015, p. 31-43.

17. Samia Kassab-Charfi et François Rastier (dir.), Mille langues et une œuvre, Paris, Éditions des archives contemporaines, coll. «Multilinguisme, traduction, création », 2016.

18. Voir Ilan Stavans, Spanglish: The Making of a New American Language, New-York, HarperCollins Publishers Inc., 2003.

19. Dans les exemples de notre contribution, nous utilisons l'italique afin de marquer l'usage de l'espagnol de sorte que le lecteur puisse identifier plus aisément l'interaction entre les différentes langues. Le gras est utilisé pour marquer d'autres langues, selon le contexte (l'italien, le portugais, le français, etc.).

20. Almuth Grésillon, « Le langage de l'ébauche : parole intérieure extériorisée », sur <http://www.persee.fr/collection/lgge > (consulté en décembre 2017), vol. XXXVI, n 147, 2002, p. 19-38.

21. Voir Olga Anokhina, « The Genesis of Texts: Planning and Interior Language », dans Belgian Journal of Linguistics, « New Approaches in Textual Linguistics », 2009, $n^{\circ} 23$, p. 63-72. 


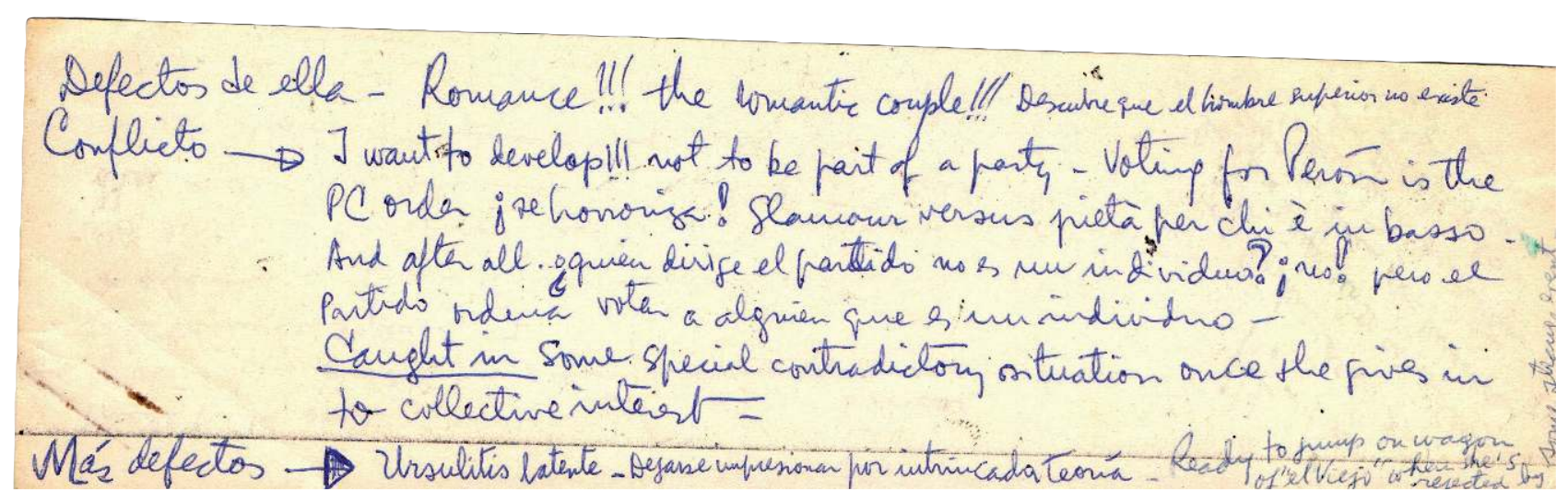

Fig. 2 : Description d'un personnage et indications métadiscursives en espagnol, anglais et italien. Notes pré-rédactionnelles du roman Pubis angelical (1979)

l'observer dans ces notes préparatoires du roman Pubis angelical (1979) :

Defectos de ella - Romance!!! The romantic couple!!! Descubre que el hombre superior no existe

Conflicto $\rightarrow$ I want to develop!!! not to be part of a party Voting for Perón is the PC order ¡se horroriza! Glamour versus pietà per chi è in basso -

And after all ¿quién dirije el partido no es un individuo? ¡no! Pero el partido ordena votar a alguien que es un individuo Caught in some special contradictory situation once she gives in to collective interest -

Más defectos $\rightarrow$ Ursulitis latente - Dejarse impresionar por intrincada teoría - Ready to jump on wagon of "el Viejo" when she's rejected by some strange event.

[Défauts d'elle - Romance!!! Le couple romantique!!! Elle découvre que l'homme supérieur n'existe pas

Conflit $\rightarrow$ Je veux développer!!! ne pas faire partie d'un parti politique - Voter pour Perón c'est l'ordre du PC pour elle c'est affreux! Glamour versus avoir de la pitié pour ceux qui sont en bas -

Et après tout, qui dirige le parti n'est pas un individu? Non! Mais le parti ordonne de voter pour quelqu'un qui est un individu -

Il est pris dans une situation particulièrement contradictoire quand il se rend à l'intérêt collectif -

Plus de défauts $\rightarrow$ Ursulitis latente - Elle se laisse impressionner par une théorie tordue - Elle est prête à sauter dans le wagon de «el Viejo» [Perón] quand elle est rejetée par un évènement étrange.]
Ainsi, il est intéressant de noter que si l'espagnol et l'anglais constituent la base du langage intérieur de Puig, il s'agit d'un idiolecte qui reste perméable aux autres langues utilisées par l'écrivain, comme c'est le cas de l'italien dans l'exemple précédent («Glamour versus pietà per chi è in basso»). De même, selon le contexte, le français et le portugais peuvent apparaître dans la phase planificatrice. Ainsi, dans les avant-textes préparatoires du scénario Felices para siempre (1980) [Joyeux pour toujours], destiné au marché cinématographique brésilien, Puig mélange le portugais avec l'espagnol, l'anglais et le français (fig. 3).

Dans certains cas, cette même matrice plurilingue se nourrit aussi d'un idiolecte familial, une langue inventée au sein de la famille Puig-Delledonne qui se caractérise par la déformation de certains mots en espagnol 22. Puig y a recours dans ses notes pour énoncer des concepts qui condensent une situation plus large, comme Locür, altération de locura (folie) dans les brouillons du roman Sangre de amor correspondido (1982), où au mélange de l'espagnol avec le portugais s'incorporent l'anglais (stranded), le français (mère parle, mariage) et l'italien (notaio) (fig. 4).

22. Cet idiolecte familial est omniprésent dans la correspondance de Puig avec sa famille. Voir Manuel Puig, Querida familia, vol. I et II. Édition, préface, notes et glossaire établis par Graciela Goldchluk, Buenos Aires, Entropía, 2006. 


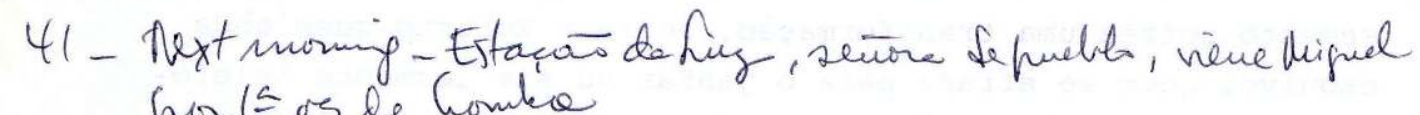

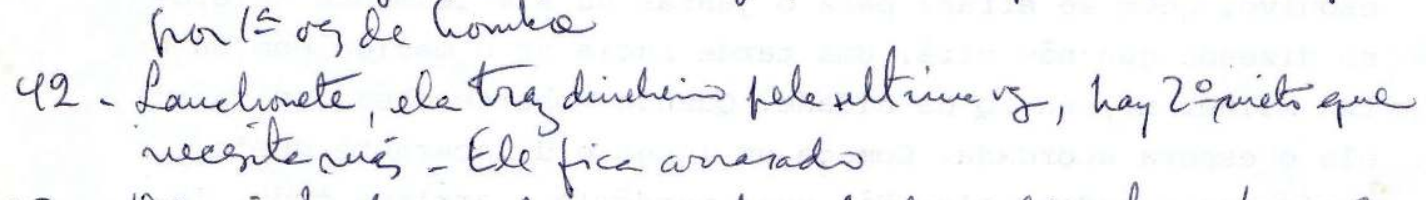

$$
\begin{aligned}
& \text { 43- Lquis do flow, ele arrasato - B. dice salira hace farre ore } \\
& \text { re ciltiviodiero }
\end{aligned}
$$

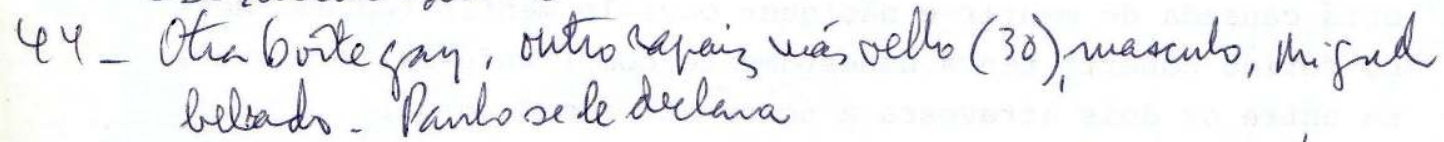

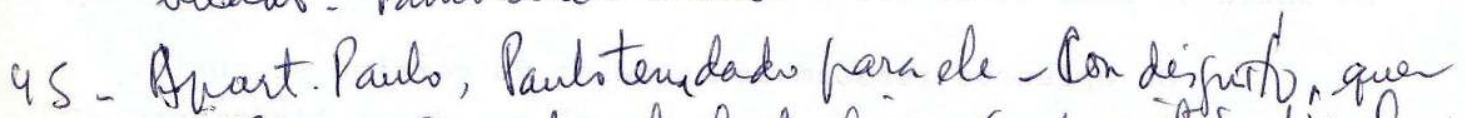

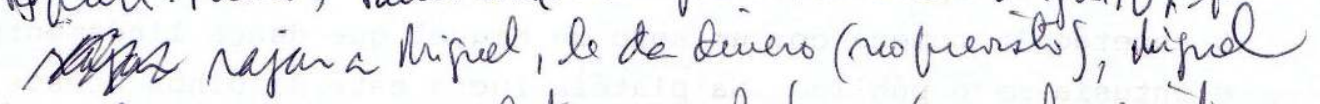

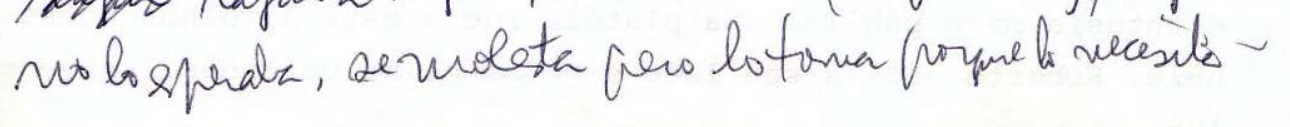

41 - Next morning - Estaçao de Luz, señora de pueblo, viene Miguel por $1^{\circ}$ vez de hombre

42 - Lanchonete, ele traz dinheiro pela ultima vez, hay $2^{\circ}$ nieto que necesita más - ele fica arrasado

43 - Depois do show, ele arrasado - B. Dice salir a hacer farra con ese último dinero

44 - Otra boîte gay, outro rapaiz mais velho (30), masculo, Miguel bebado - Pancho se le declara

[41 - Le matin suivant - Gare de Luz, une villageoise, Miguel vient pour la lre fois en tant qu'homme

42 - Bar, il amène de l'argent pour la dernière fois, il y a un $2^{e}$ petit-fils qui en a plus besoin - il reste dévasté

43 - Après le spectacle, lui dévasté - B. propose d'aller faire la fête avec ce dernier argent

44 - Une autre boîte gay, un autre garçon plus jeune (30), masculin, Miguel saoul, Pancho lui déclare son amour]

Fig. 3 : Planification des scènes du scénario pour le cinéma Felices para siempre (c. 1980)

Outre le mélange improbable et totalement aléatoire de plusieurs langues dans ces notes planificatrices - aléatoire dans le sens où il n'existe aucune explication rationnelle de la manière et du fait même de convoquer toutes ces langues sinon la logique interne de l'écrivain - il est intéressant de relever l'influence du dialecte Parma-Piacenza parlé par la famille Puig qui consiste, d'une part, à transformer la voyelle - u - de l'espagnol en - ü - dialectale (qui se prononce alors comme le «u» français) $: \mathbf{u} \rightarrow$ ü. D'autre part, selon un autre trait phonologique particulièrement marqué des dialectes du nord de l'Italie, elle amène à la chute de la voyelle finale du mot : $\mathrm{a} \rightarrow \emptyset$. Or, ici ces particularités phonologiques se trouvent appliquées au mot espagnol locura en le transformant en Locür, le tout complexifiant encore davantage ce qu' on pourrait appeler l'idiolecte de l'écrivain ou son langage intérieur.

Comme nous l'avons signalé, l'usage de ce langage intérieur, forgé à partir des langues maîtrisées par l'écrivain, se perçoit plutôt dans la phase pré-rédactionnelle et ses traces s'effacent dans les phases d'écriture postérieures.

\section{L'écriture en deux langues à l'étape rédactionnelle}

Lors de la textualisation, pour certaines œuvres, on peut voir Puig utiliser une autre stratégie plurilingue : l'autotraduction. En effet, si pour la plupart des écrivains plurilingues, quand il s'agit de faire deux œuvres séparées, l'autotraduction à proprement parler vient souvent une fois l'œuvre - ou le premier jet de l'œuvre - terminée, chez Puig on trouve des éléments d'autotraduction en pleine phase rédactionnelle au point de ne pas pouvoir toujours distinguer si l'autotraduction a été faite postérieurement ou pratiquement en même temps.

De ce point de vue, l'étude du roman Maldición eterna a quien lea estas páginas [Malédiction éternelle à qui lira ces pages], souvent déclaré par les spécialistes comme un texte autotraduit par Puig de l'anglais vers l'espagnol, représente un intérêt particulier. L'auteur ne s'est jamais prononcé au sujet de l'ordre dans lequel la version espagnole et la version anglaise du roman ont été créées. Or la notice qui accompagne la publication de la version anglaise du roman, 


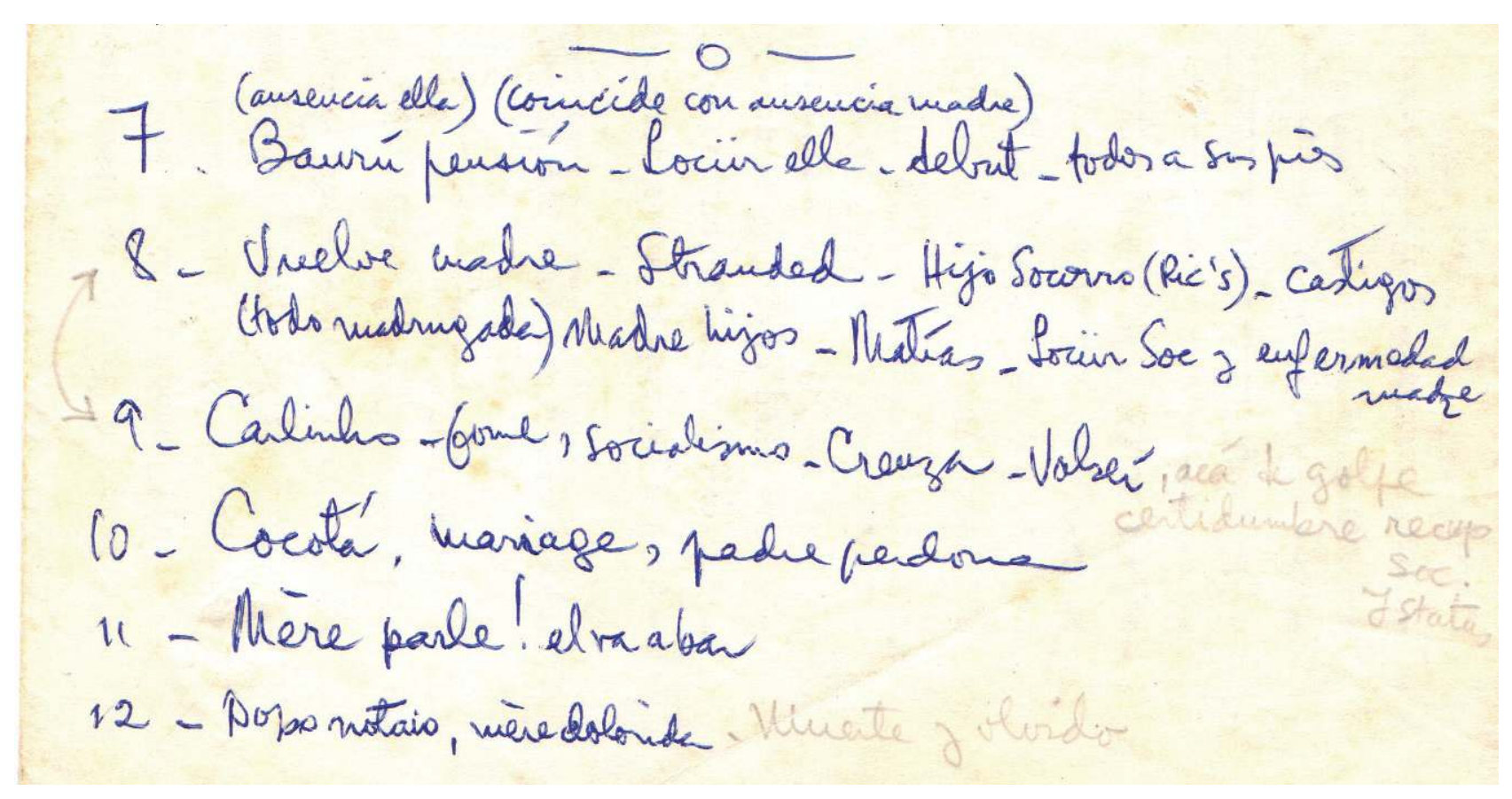

7 - (ausencia ella) (coincide con ausencia madre)

Baurú pensión - Locür ella - debut - todos [illis.]

8 - Vuelve madre - Stranded - Hijo socorro (Ric's) - castigos (todo madrugada) madre hijos - Matías - Locür Soc y enfermedad madre

9 - Carlinho - fome, socialismo - Creuza - Valseí [- acá de golpe recup soc. y status]

10 - Cocotá, mariage, padre perdona

11 - Mère parle! Él va a bar

12 - Papa notaio, mère dolorida [-Muerte y olvido]

[7 - (absence d'elle) (coüncide avec absence mère)

Baurú motel - Locür elle - début - tous [illis.]

8 - mère revient - bloquée - fils au secours (Ric's) - punitions (tout à l'aube) mère fils - Matías - Locür Soc et maladie mère

9 - Carlinho - faim, socialisme - Creuza - Valseí [-ici, d'un coup, récup. soc et statut]

10 - Cocotá, mariage, père pardonne

11 - Mère parle! il va au bar

12 - Père notaire, mère souffre [-Mort et oubli]

Fig. 4 : Notes de planification pour le roman Sangre de amor correspondido (1982)

parue deux ans après sa publication en espagnol, rend les choses encore plus confuses : «Originally published as a translation into Spanish by Seix Barral, 1980» («Roman publié originellement comme une traduction en espagnol par Seix Barral, $1980 »)$. Généralement, les brouillons permettent de lever ce genre de doute ${ }^{23}$. Cependant, les états génétiques du roman se révèlent si complexes qu'il n'est pas toujours facile d'identifier si l'écriture en espagnol est antérieure, postérieure ou simultanée à celle en anglais comme nous allons le montrer à présent.

Grâce à une observation minutieuse des modifications, on peut tenter d'établir quelle version a été initiale et a entraîné les changements dans une deuxième version écrite dans une autre langue. Notre étude des brouillons dactylographiés en deux langues montre que la version espagnole a probablement été écrite en premier. Ensuite, Puig semble l'avoir traduite vers l'anglais en la tapant directement à la machine et en y apportant un certain

23. Ainsi, face aux déclarations contradictoires de J. J. Rabearivelo quant à l'ordre d'écriture de ces poèmes en français et en malgache, Claire Riffard a pu identifier une véritable stratégie créative qui a guidé la création de l'œuvre bilingue du poète. Voir Claire Riffard, « Étude des manuscrits malgaches bilingues de J. J. Rabearivelo », dans Olga Anokhina (dir.), Multilinguisme et créativité littéraire, Louvain-la-Neuve, Academia Bruylant/L'Harmattan, 2012, p. 55-65. 


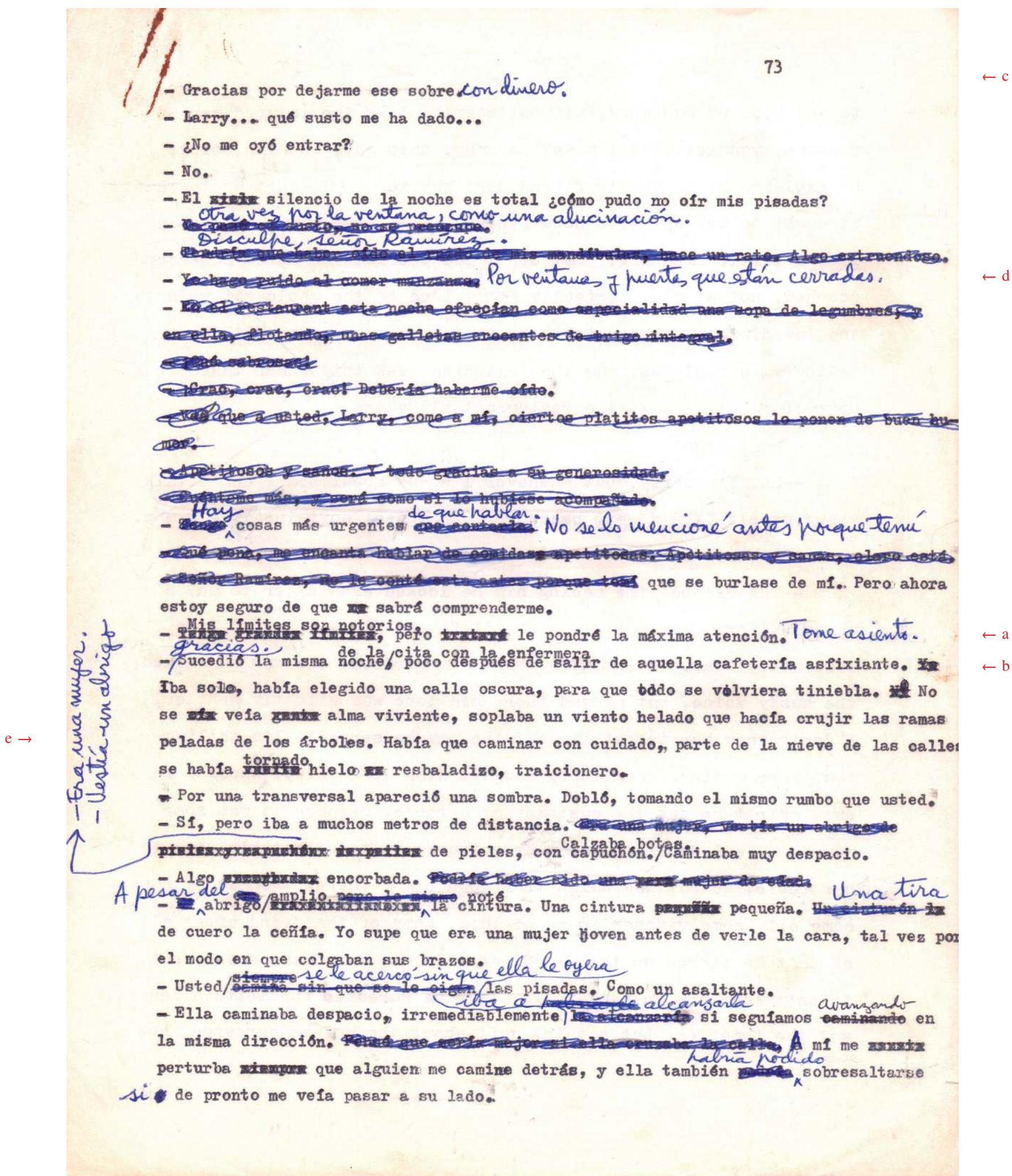

Fig. 5 : Dactylographie avec corrections manuscrites en espagnol de Maldición eterna a quien lea estas páginas (1980) 


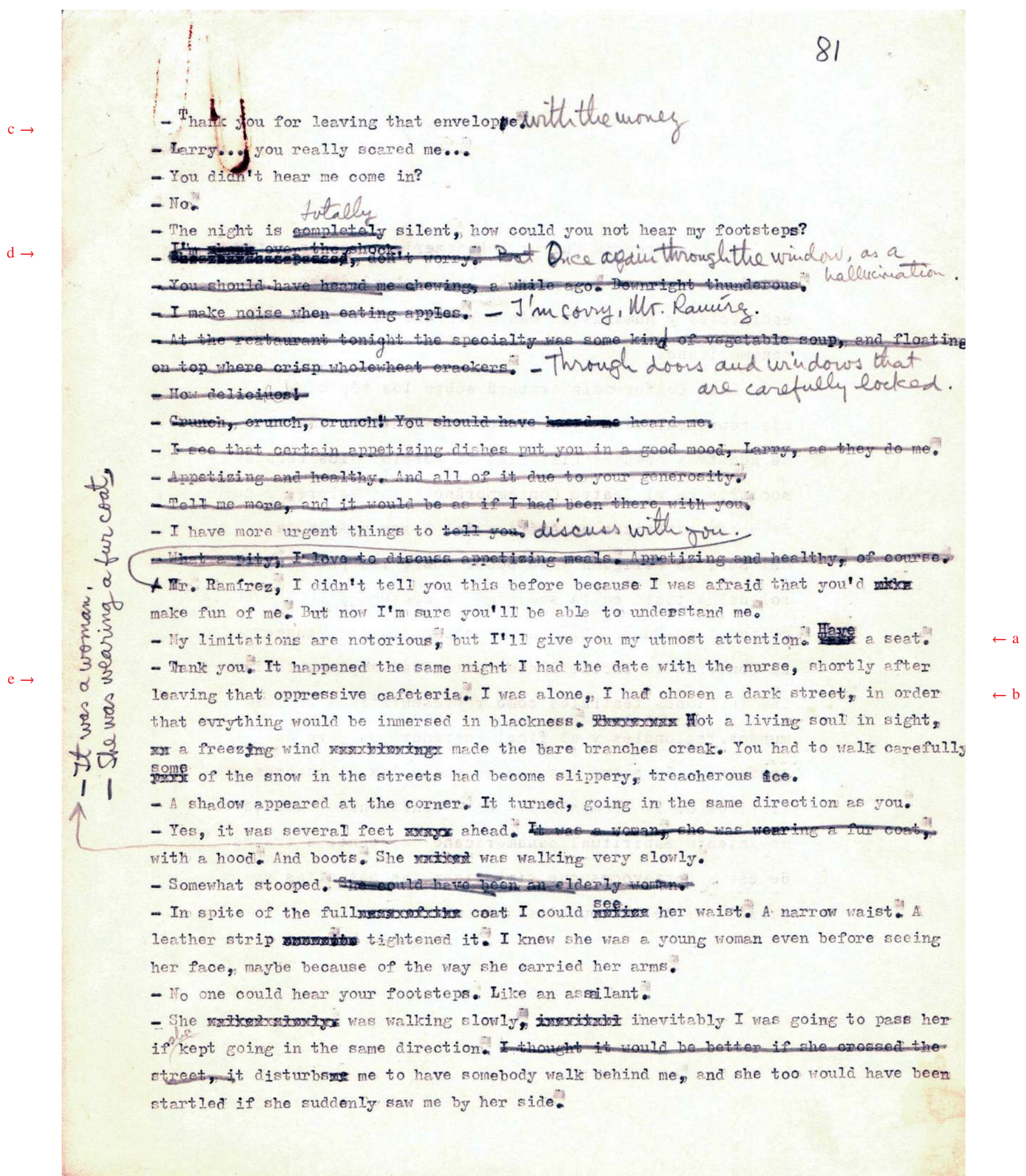

Fig. 6 : Dactylographie avec corrections manuscrites en anglais de Maldición eterna a quien lea estas páginas (1980) 


\begin{tabular}{|c|c|}
\hline DACTYLOGRAPHIE EN ANGLAIS* & DACTYLOGRAPHIE EN ESPAGNOL*** \\
\hline $\begin{array}{l}\text {-My limitations are notorious, but I'll give you my outmost } \\
\text { attention. Take Have a seat. } \quad \mathrm{a} \rightarrow\end{array}$ & $\begin{array}{l}\text { - Tengo grandes límites Mis límites son notorios pero trataré le } \\
\text { pondré la máxima atención. Tome asiento. } \quad \mathrm{a} \rightarrow\end{array}$ \\
\hline $\begin{array}{l}\text { - Thank you. It happened the same night I had the date with } \\
\text { the nurse, shortly after leaving that oppresive cafeteria. } \quad \mathrm{b} \rightarrow\end{array}$ & $\begin{array}{l}\text { _Gracias. Sucedió la misma noche de la cita con la enfermera, poco } \\
\text { después de salir de aquella cafetería asfixiante. } \mathrm{b} \rightarrow\end{array}$ \\
\hline
\end{tabular}

Tableau 1

\begin{tabular}{|c|c|}
\hline DACTYLOGRAPHIE EN ESPAGNOL & DACTYLOGRAPHIE EN ANGLAIS \\
\hline -Gracias por dejarme ese sobre: con dinero. $\quad \mathrm{c} \rightarrow$ & Thank you for leaving that enveloppe.with the money $\mathrm{c} \rightarrow$ \\
\hline $\begin{array}{l}\text {-Ya pasó el susto, no se preocupe. Otra vez por la ventana, } \\
\text { como una alucinación } \quad \mathrm{d} \rightarrow\end{array}$ & $\begin{array}{l}\text { The shock has passed F'm over the shoek, don't worry. But } \\
\text { Once again through the window, as a hallucination. } \quad \mathrm{d} \rightarrow\end{array}$ \\
\hline $\begin{array}{l}\text {-Sí, pero iba a muchos metros de distancia. Era una mujer, } \\
\text { vestía un abrigo de pieles y capuchón de peiles, / —Era una } \\
\text { mujer. -Vestía un abrigo / de pieles e } \rightarrow\end{array}$ & $\begin{array}{l}\text {-Yes, it was aseveral feet away ahead. It was a woman, se } \\
\text { she was wearing a fur coat, /LIt was a woman. - She was } \\
\text { wearing a fur coat,/ } \mathrm{e} \rightarrow\end{array}$ \\
\hline
\end{tabular}

Tableau 2

(*) Document N.F. 21 du dossier « Pre-textos redaccionales. Español. Bloque 1 », p. 73. Dans les exemples qui suivent, l'italique indique les ajouts manuscrits. - $(* *)$ Document N.F. 55 du dossier « Pre-textos redaccionales. Inglés. Bloque 11 », p. 81. Les lettres fléchées renvoient aux figures 5 et 6 à la page précédente.

nombre de modifications. Le texte en anglais est donc très proche de l'espagnol mais il comporte un certain nombre d'ajouts ou de modifications faits au fil de la traduction. Après cela, l'écrivain s'est employé à rapporter toutes ces modifications, qui sont directement intégrées dans la dactylographie en anglais, à la version déjà existante en espagnol. Ces modifications sont faites soit sous forme dactylographiée interlinéaire (Mis limites son notorios [My limitations are notorious], Calzaba botas [And boots]), soit elles sont transcrites manuellement au stylo (Gracias [Thank you], A pesar de [In spite of]) (tableau 1).

Une fois ces deux textes fixés, Puig fait une campagne de relecture lors de laquelle il corrige les deux textes de manière identique (tableau 2).

Un ajout identique dans la marge gauche sur les deux versions vient confirmer que la campagne de correction - au crayon sur la version anglaise et au stylo-bille bleu sur la version espagnole - pourrait avoir lieu au même moment de façon simultanée :

- It was a woman. She was wearing a fur coat.

— Era una mujer. Vestía un abrigo de pieles.
[-C'était une femme.

— Elle portait un manteau en fourrure.]

Cet ajout manuscrit marginal dans les deux langues, opéré lors d'une campagne de relecture finale, faisait à l'origine partie des versions textuelles dactylographiées en deux langues et constitue, par conséquent, une «rature de transfert 24 ».

Le caractère strictement identique des modifications apportées manuellement laisse penser en effet que les deux versions - espagnole et anglaise - auraient pu être corrigées simultanément, malgré la différence d'instrument utilisé (le crayon pour la version anglaise et le stylo bleu pour le texte en espagnol).

Toutefois, toutes les corrections ne sont pas identiques. L'exemple suivant montre l'influence de l'espagnol qui opère lors d'une campagne de relecture sur les corrections

24. Pierre-Marc de Biasi, « Qu'est-ce qu'une rature ? », dans Bertrand Rougé (dir.), Ratures et Repentirs ( $5^{\mathrm{e}}$ colloque du CICADA, université de Pau, 1-3 décembre 1994), Pau, Publications de l'université de Pau, 1996, p. 17-47. 


\begin{tabular}{|l|l|}
\hline DACTYLOGRAPHIE EN ANGLAIS & DACTYLOGRAPHIE EN ESPAGNOL \\
\hline $\begin{array}{l}\text { There was Not a living soul in a sight, a freezying wind was } \\
\text { blowing made the branches creak. You had to walk carefully } \\
\text { some of the snow in the streets had become slippery, } \\
\text { treacherous ice. }\end{array}$ & $\begin{array}{l}\text { No se eía veía gente alma viviente, soplaba un viento helado } \\
\text { que crujir las ramas peladas de los árboles. Había que } \\
\text { caminar con cuidado, parte de la nieve de las calles se había } \\
\text { vuelte tornado hielo resbaladizo, traicionero. }\end{array}$ \\
\hline
\end{tabular}

de la version anglaise : on voit ainsi l'adverbe «totally» [«totalement»] qui est ajouté à la main dans le texte en anglais en remplacement de l'adverbe «completely» [«complètement»] :

— The night is eompletely totally silent, how could you not hear my footsteps?

[-La nuit est complètement totalement silencieuse, comment avez-vous pu ne pas entendre mes pas?]

Cette autre modification effectuée au crayon et en interlinéaire au moment de la relecture est sans aucun doute faite sous l'influence de la version espagnole, écrite directement à la machine :

—El sielen silencio de la noche es total ¿ cómo pudo no oír mis pisadas?

[-Le silence de la nuit est total comment n'avez-vous pas entendu mes pas?]

Pour résumer, le texte dactylographié en espagnol constitue un état génétique 1. Le texte dactylographié en anglais est un état génétique 2. Les corrections dactylographiées et interlinéaires sur le texte espagnol composent un état génétique 3 et enfin, les corrections manuelles identiques reportées sur la version anglaise et la version espagnole forment un état génétique 4 double : $4 \mathrm{a}$ - en espagnol et $4 b-$ en anglais. Cet exemple montre parfaitement la manière circulaire dont fonctionne l'autotraduction.

Cependant, cette vision est ébranlée par l'extrait présenté dans le tableau 3.

Si nous suivons les corrections qui ont donné lieu à la phrase «No se veía alma viviente» [On ne voyait pas une seule âme vivante] nous pouvons constater que Puig remplace la phrase «No se veía gente» [On ne voyait personne], par «alma viviente» [âme vivante], qui ressemble à un calque d'une expression idiomatique de la version anglaise (not a living soul). Cette construction est très rare voire incorrecte en espagnol. Dans ce cas, c'est la langue anglaise qui semble influencer la version espagnole. Mais regardons le verbe qui suit cette même phrase : «soplaba» [soufflait]. Ce verbe est écrit directement à la machine en espagnol, alors que, dans la dactylographie en anglais, son équivalent «was blowing » est barré, sans que cela empêche la continuation de la phrase. On voit donc que, d'une part, l'espagnol semble être légèrement antérieur à l'anglais car, cette fois-ci c'est le verbe espagnol «soplar» qui inspire clairement un calque lexical en anglais «blow». D' autre part, le calque est supprimé immédiatement sans que cela empêche le développement de la phrase et la saisie dactylographique continue. On semble donc être en présence d'une écriture quasi simultanée en deux langues, ce qui est très rare pour l'écriture en prose. Le problème est que les deux versions sont dactylographiées. Or il semblerait que Puig n'avait qu'une seule machine à écrire en sa possession (probablement la machine avec un clavier espagnol mais qui permettait également d'écrire en anglais), ce qui exclut la possibilité d'écriture véritablement «simultanée». En revanche, il ne faut pas négliger le fait qu'une personne plurilingue, de surcroît écrivant en deux langues peut faire des interférences linguistiques. De ce fait, une expression courante qui semble influencer l'écriture dans une autre langue, comme c'est le cas avec No se veía alma viviente [There was Not a living soul in a sight], pourrait simplement être un calque formé dans l'esprit de l'écrivain et couché sur le papier...

\section{Conclusion}

Comme nous avons pu l'observer dans cette étude, la création pour Puig est toujours un processus traversé par des réappropriations linguistiques multiples. Pendant la phase de planification de ses textes, on voit à l'œuvre un 
phénomène qui ressemble au langage intérieur, incohérent en apparence, tel qu'il a été défini dans les travaux pionniers de Lev Vygotski25. La particularité de ce langage, manifeste dans les plans de Puig, consiste dans le fait qu'il est construit à partir d'un substrat plurilingue qui contient des éléments provenant essentiellement de l'anglais et de l'espagnol mais aussi de l'italien, du portugais du Brésil et d'un dialecte italien.

Lors de l'étape de textualisation, surtout dans les œuvres que Puig envisage de publier à la fois en espagnol et en anglais (comme Maldición eterna a quien lea estas páginas), le mélange des langues disparaît en laissant la place à l'écriture de deux textes monolingues en deux langues différentes. Même si les codes linguistiques semblent strictement séparés à cette étape, le travail d'écriture et surtout celui de correction simultanée montrent l'existence de liens étroits entre deux langues, faisant dialoguer en permanence deux textes in statu nascendi.
Les généticiens ont montré que les écrivains «à structuration rédactionnelle», comme Proust, ont parfois besoin de planifier et que, inversement, les «planificateurs » abandonnent à l'occasion le programme préétabli en se laissant mener par l'écriture sans planification préalable; de même, le mode d'écriture d'un écrivain plurilingue ne se réduit pas nécessairement à une seule stratégie scripturaire, à une seule forme de plurilinguisme. Il peut varier avec le temps ou prendre des aspects divers aux différents stades de l'élaboration de l'œuvre. C'est ce que l'étude des documents de travail de Manuel Puig nous a permis d'observer, alors même que les textes publiés ne laissent rien apercevoir du complexe et passionnant tissage des langues qui y est à l'œuvre.

25. Lev Vygotski, Pensée et Langage, Paris, La Dispute, 1997 [1934]. 
Delfina Cabrera est docteur en lettres (université de Perpignan/université de Bergame) et chercheur au Département d'études de critique génétique et d'archives d'écrivains de l'université de La Plata, Argentine. Ses recherches portent sur les rapports entre la traduction, le plurilinguisme et la création dans la littérature latino-américaine. Elle est l'auteur de l'ouvrage Las lenguas vivas. Zonas de exilio y traducción en Manuel Puig (2016), ainsi que de nombreuses traductions vers l'espagnol.

delfinacabrera@gmail.com

\section{Tisser le texte et cacher les fils : l'écriture plurilingue de Manuel Puig}

La production littéraire de l'écrivain argentin Manuel Puig (19321990) suscite depuis quelques années un grand intérêt. Néanmoins, l'attention de la critique s'est concentrée essentiellement sur certaines caractéristiques de son œuvre, comme la déstabilisation des genres narratifs, la tension entre la culture des élites et la culture populaire, l'absence de narrateur omniscient, les questions de genre, en laissant inexplorés son plurilinguisme et ses pratiques de traduction. Pour pallier l'absence d'intérêt pour la dimension plurilingue de ses écrits, notre contribution offrira un aperçu de l'usage des langues étrangères dans le processus créatif de Puig. L'étude génétique des documents de travail plurilingues de Puig ouvre en effet la voie à de nouvelles interprétations de son œuvre, en permettant d'aller au-delà de l'image canonisée de l'auteur en tant qu'écrivain latino-américain «monolingue».

For the last few years the literary production of the Argentine writer Manuel Puig (1932-1990) has sparked much interest. However, criticism has essentially focused on certain characteristics of his works, such as the destabilization of narrative genres, the tension between elite and popular cultures, the absence of an omniscient narrator, and questions of genre. It left unexplored his multilingualism and his translation techniques. To compensate for this absence of interest in the multilingual dimension of his works, we will show how foreign languages are used in Puig's creative processes. The genetic study of his multilingual working documents pave the way for new interpretations of his works, to go beyond the canonized image of the author as a Latino-American "monolingual" writer.

Die literarische Produktion des argentinischen Schriftstellers Manuel Puig (1932-1990) erfährt seit einigen Jahren großes Interesse. Dieses konzentrierte sich jedoch vor allem auf bestimmte Merkmale seiner Arbeit, wie die Destabilisierung der Erzählgenres, die Spannung zwischen Elitekultur und Populärkultur, die Abwesenheit des allwissenden Erzählers sowie die Fragen nach dem Genre, und ließ seine Mehrsprachigkeit und seine Übersetzungspraktiken unerforscht. Um dem mangelnden Interesse an der mehrsprachigen Dimension seiner Schriften zu begegnen, wird unser Beitrag einen Einblick in den Gebrauch von Fremdsprachen in Puigs Schaffensprozess geben. Die genetische Untersuchung von Puigs mehrsprachigen Arbeitsdokumenten öffnet den Weg zu neuen Interpretationen seiner Arbeit, die es erlauben, über das kanonisierte Bild des Autors als eines ,einsprachigen" lateinamerikanischen Schriftstellers hinauszugehen.
La producción literaria del escritor argentino Manuel Puig (1932-1990) suscita desde años un gran interés crítico. Sin embargo, la atención de los especialistas se ha centrado principalmente en algunas características de su obra, como la desestabilización de los géneros narrativos, la tensión entre la cultura letrada y la cultura popular, la ausencia de un narrador omnisciente o las cuestiones de género, y ha dejado en los márgenes el estudio del plurilingüismo y las prácticas de traducción de Puig. Para compensar esta falta de interés en la dimensión plurilingüe de su escritura, este artículo ofrece un análisis del uso de las lenguas extranjeras en el proceso creativo del autor. El estudio genético de los documentos de trabajo plurilingües de Puig abre el camino a nuevas interpretaciones de su obra, al tiempo que permite ir más allá de la imagen canonizada de Puig como escritor latinoamericano "monolingüe".

A produção literária do escritor argentino Manuel Puig (19321990) tem suscitado grande interesse nos últimos anos. No entanto, a atenção da crítica concentra-se em características como a desestabilização dos géneros narrativos, a tensão entre a cultura das elites e a cultura popular, a ausência do narrador omnisciente e a questão do género, passando despercebidas a questão do seu plurilinguismo e as suas práticas de tradução. Para suprir essa falha, este estudo oferece uma visão geral da utilização de línguas estrangeiras no processo criativo de Puig. A análise genética de materiais plurilingues de Puig possibilita novas interpretações da sua obra, que transcendem a imagem estabelecida do autor como um escritor latino-americano "monolingue".

La produzione letteraria dello scrittore argentino Manuel Puig (1932-1990) suscita da qualche anno un grande interesse. L'attenzione della critica si è tuttavia concentrata essenzialmente su alcune caratteristiche della sua opera, quali la destabilizzazione dei generi narrativi, la tensione tra cultura d'elite e cultura popolare, l'assenza del narratore onnisciente, le questioni di genere, lasciando inesplorato il plurilinguismo e le pratiche di traduzione. Per rimediare a questa mancanza d'interesse per la dimensione plurilingue dei suoi scritti, l'articolo traccerà un quadro dell'uso delle lingue straniere nel processo creativo dell'autore. Lo studio genetico dei suoi documenti di lavoro plurilingue apre la strada a nuove interpretazioni della sua opera, permettendo così di superare l'immagine canonizzata di Puig come scrittore sudamericano "monolingue". 